\title{
MRI-Based Classification Models in Prediction of Mild Cognitive Impairment and Dementia in Late-Life Depression
}

\author{
Aleksandra K. Lebedeva ${ }^{1 *}$, Eric Westman', Tom Borza ${ }^{2,3}$, Mona K. Beyer ${ }^{4}$, \\ Knut Engedal|5,6, Dag Aarsland ${ }^{1,7,8}$, Geir Selbaek ${ }^{2,3,5,6}$ and \\ Asta K. Haberg ${ }^{9,10}$ for the Alzheimer's Disease Neuroimaging Initiative ${ }^{\dagger}$
}

${ }^{1}$ Department of Neurobiology, Care Sciences and Society, Karolinska Institutet, Stockholm, Sweden, ${ }^{2}$ Centre for Old Age Psychiatric Research, Innlandet Hospital Trust, Brumunddal, Norway, ${ }^{3}$ Institute of Clinical Medicine, Faculty of Medicine, University of Oslo, Oslo, Norway, ${ }^{4}$ Department of Radiology and Nuclear Medicine, Oslo University Hospital, Rikshospitalet, Oslo, Norway, ${ }^{5}$ Department of Geriatric Medicine, Oslo University Hospital, Tønsberg, Norway, ${ }^{6}$ Oslo and Norwegian National Advisory Unit for Aging and Health, Tønsberg, Norway, ${ }^{7}$ Department of Old Age Psychiatry, Institute of Psychiatry, Psychology and Neuroscience, King's College London, London, UK, ${ }^{8}$ Center for Age-Related Medicine, Stavanger University Hospital, Stavanger, Norway, ${ }^{9}$ Department of Neuroscience, Norwegian University of Science and Technology, Trondheim, Norway, ${ }^{10}$ Department of Radiology and Nuclear Medicine, St. Olav's University Hospital, Trondheim, Norway

\section{OPEN ACCESS}

Edited by: Lia Fernandes,

University of Porto, Portugal

Reviewed by:

Douglas Watt,

Quincy Medical Center and Cambridge Health Alliance, USA Ramesh Kandimalla,

Texas Tech University, USA

*Correspondence:

Aleksandra K. Lebedeva aleksandra.ki.lebedeva@gmail.com

Received: 31 October 2016 Accepted: 17 January 2017

Published: 02 February 2017

Citation: Lebedeva AK, Westman E, Borza T, Beyer MK, Engedal K, Aarsland D, Selbaek G and Haberg AK (2017) MRI-Based Classification Models

in Prediction of Mild Cognitive Impairment and Dementia in Late-Life

Depression

Front. Aging Neurosci. 9:13. doi: 10.3389/fnagi.2017.00013
Objective: Late-life depression (LLD) is associated with development of different types of dementia. Identification of LLD patients, who will develop cognitive decline, i.e., the early stage of dementia would help to implement interventions earlier. The purpose of this study was to assess whether structural brain magnetic resonance imaging (MRI) in LLD patients can predict mild cognitive impairment (MCl) or dementia 1 year prior to the diagnosis.

Methods: LLD patients underwent brain MRI at baseline and repeated clinical assessment after 1-year. Structural brain measurements were obtained using Freesurfer software (v. 5.1) from the T1W brain MRI images. MRI-based Random Forest classifier was used to discriminate between LLD who developed $\mathrm{MCl}$ or dementia after 1-year follow-up and cognitively stable LLD. Additionally, a previously established Random Forest model trained on 185 patients with Alzheimer's disease (AD) vs. 225 cognitively normal elderly from the Alzheimer's disease Neuroimaging Initiative was tested on the LLD data set (ADNI model).

Results: $\mathrm{MCl}$ and dementia diagnoses were predicted in LLD patients with 76\%/68\%/84\% accuracy/sensitivity/specificity. Adding the baseline Mini-Mental State Examination (MMSE) scores to the models improved accuracy/sensitivity/specificity to $81 \% / 75 \% / 86 \%$. The best model predicted $\mathrm{MCl}$ status alone using $\mathrm{MRI}$ and baseline MMSE scores with accuracy/sensitivity/specificity of $89 \% / 85 \% / 90 \%$. The most important region for all the models was right ventral diencephalon, including

\footnotetext{
Abbreviations: CC, corpus callosum; CTH, cortical thickness; LLD, late-life depression; RF, random forest method; R-HC right hippocampus; R-VD, right ventral diencephalon; SV, subcortical volumetric measurement.

'Data used in preparation of this article were obtained from the Alzheimer's Disease Neuroimaging Initiative (ADNI) database (adni.loni.usc.edu). As such, the investigators within the ADNI contributed to the design and implementation of ADNI and/or provided data but did not participate in analysis or writing of this report. A complete listing of ADNI investigators can be found at: http://adni.loni.usc.edu/wp-content/uploads/ how_to_apply/ADNI_Acknowledgement_List.pdf
} 
hypothalamus. Its volume correlated negatively with the number of depressive episodes. ADNI model trained on AD vs. Controls using SV could predict MCI-DEM patients with 67\% accuracy.

Conclusion: LDD patients developing $\mathrm{MCl}$ and dementia can be discriminated from LLD patients remaining cognitively stable with good accuracy based on baseline structural MRI alone. Baseline MMSE score improves prediction accuracy. Ventral diencephalon, including the hypothalamus might play an important role in preservation of cognitive functions in LLD.

Keywords: depression, $\mathrm{MCl}$, Alzheimer's disease, Freesurfer, neurodegeneration, hypothalamus, ventral diencephalon, depressive episodes

\section{INTRODUCTION}

Depression is associated with accelerated brain aging (Simon et al., 2006; McKinney and Sibille, 2013; Koutsouleris et al., 2014), and is a risk factor for different types of dementia (Aziz and Steffens, 2013; Diniz et al., 2013; Byers and Yaffe, 2014; Cooper et al., 2015; Mourao et al., 2015).

Unfortunately, early identification of predementia states in people with LLD is challenging as reduced cognitive scores can be confounded by the depressive state. Thus depression is an exclusion criterion for some definitions of mild cognitive impairment (MCI; Steffens et al., 2006). It is challenging to predict whether cognitive impairment identified during a depressive episode in a LLD patient will improve after treatment of depression or will progress to dementia. However, it is known that among seniors with MCI identified during a depressive episode only $17 \%$ experienced cognitive improvement after 2 years of follow-up (Steffens et al., 2009). Early identification of those LLD patients with increased risk of progressive cognitive decline could allow for more targeted clinical actions, for instance choice of antidepressant (du Jardin et al., 2016; García-Fuster and García-Sevilla, 2016) or other interventions, (Ngandu et al., 2015) and possibly neuroprotective drugs in the future.

Biomarkers known from Alzheimer's disease (AD) research may be used to identify a neurodegenerative process and/or increased risk of developing dementia in LLD patients in particular during a depressive episode when neurocognitive functions may not be reliably assessed. The biomarkers include amyloid-beta positron emission tomography (PET) brain imaging, magnetic resonance imaging (MRI) based cortical and subcortical structural measurements, cerebrospinal fluid tau, and amyloid-beta levels (American Psychiatric Association, 2013; Dickerson and Wolk, 2013). However, it is still not known if these biomarkers can be utilized to identify or predict future cognitive impairment (MCI or dementia status) in LLD.

The aims of this study were: (1) To assess whether structural T1 weighted (T1W) 3D brain MRI obtained during the depressive episode in LLD patients can discriminate LLD who were diagnosed with MCI or dementia after 1-year follow-up from cognitively stable LLD using Random Forest classifier. (2) To identify which regions are affected in LLD with subsequent cognitive impairment. (3) To evaluate the feasibility of using biomarkers derived from $\mathrm{AD}$ research, by implementing a classifier trained on structural 3D brain MRI from the Alzheimer's Disease Neuroimaging Initiative (ADNI) sample.

\section{MATERIALS AND METHODS}

\section{Cohorts}

Two cohorts were used in this study:

(1) PRODE (Prognosis of depression in the elderly) - was used as the main cohort (Borza et al., 2015). The PRODE study is a prospective multicenter study including 169 patients $\geq 60$ years referred to treatment for depression at nine centers of geriatric psychiatry in Norway (Borza et al., 2015). Exclusion criteria were life threatening diseases and severe aphasia (as it would reduce the validity of the neuropsychological assessment). Clinical and neuropsychological data were collected using standardized clinical, psychiatric, and neuropsychological assessment (see below). Of the 169 patients, 126 underwent brain MRI examination at inclusion (Lebedeva et al., 2015) T1W 3D brain MRI images were available for 112 patients (Figure 1).

(2) ADNI - An established classification model based on RF trained to discriminate between patients with $\mathrm{AD}(n=185)$ and healthy controls (HC, $n=225$ ) using Freesurfer (v. 5.1) measurements from T1W 3D brain MRI was also used on the PRODE dataset. The training data was obtained from the ADNI database ${ }^{1}$. The ADNI was launched in 2003 as a public-private partnership, led by Principal Investigator Michael W. Weiner, MD. The primary goal of ADNI has been to test whether serial MRI, PET, other biological markers, and clinical and neuropsychological assessment can be combined to measure the progression of $\mathrm{MCI}$ and early $\mathrm{AD}$. AD patients met the NINCDS/ADRDA criteria for probable AD. The T1W 3D MRI-Freesurfer based model had AD vs. HC accuracy of $90 \%$ for the testing dataset and predicted conversion in additional sample of MCI $(n=165)$ to AD with accuracy $79 \%$. The most important regions for the discrimination were: left and right hippocampi, left amygdala, left and right entorhinal cortices, and left inferior temporal cortex (Lebedev et al., 2014). We hypothesized that this model could be used to predict $\mathrm{MCI} / \mathrm{AD}$ conversion in the PRODE cohort.

\footnotetext{
${ }^{1}$ adni.loni.usc.edu
} 


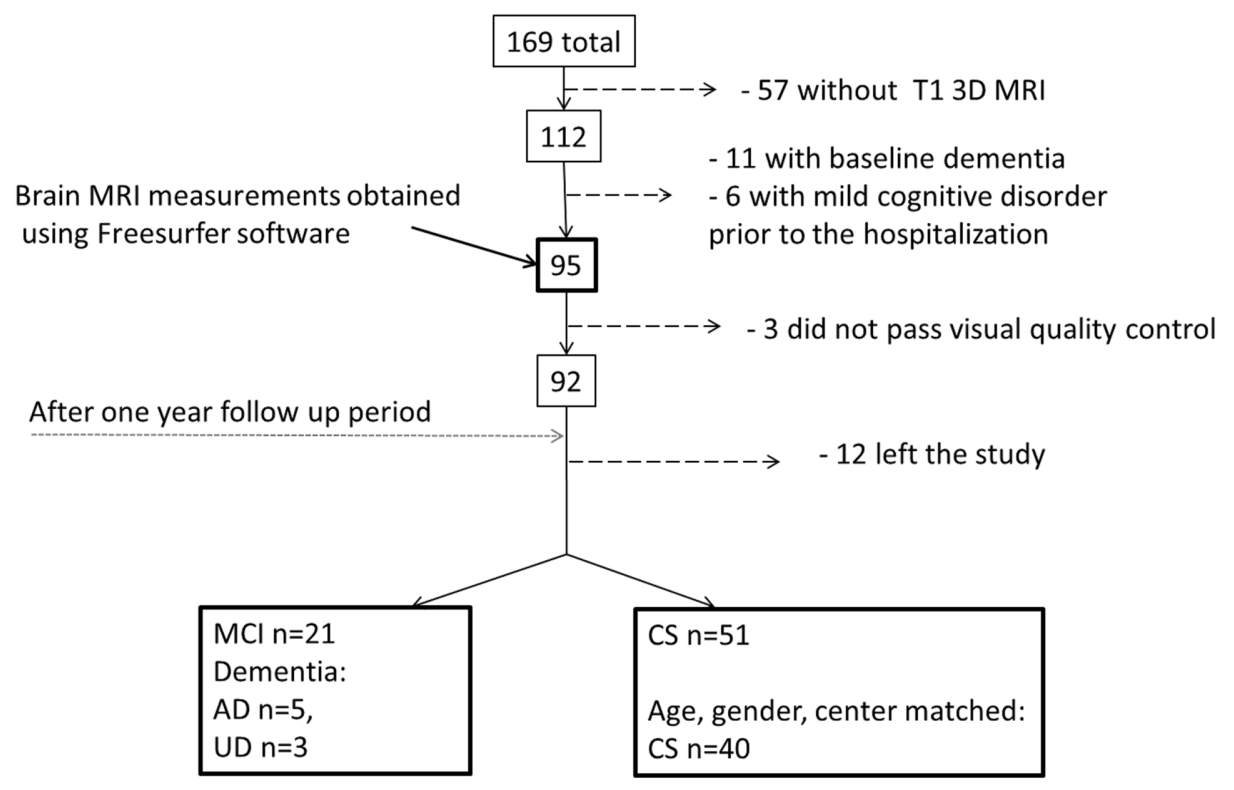

FIGURE 1 | Flow chart describing patient selection and attrition from admission to 1-year follow-up assessment. AD, Alzheimer's disease; UD, Unspecified dementia; MCl, mild cognitive impairment; CS, cognitively stable control group; mild cognitive disorder - F 06.7 (ICD-10).

\section{Clinical and Neuropsychological Assessment}

Psychiatric evaluation and neuropsychological assessment were performed three times: at admission to the department of geriatric psychiatry (baseline) and discharge, and after 1year. At each participating center trained clinicians performed the psychiatric and neuropsychological assessments using a harmonized procedure and the same protocol (Borza et al., 2015).

Clinical assessments included: demographic information, history of depression or other psychiatric condition, family history of psychiatric problems, current psychiatric problems, and treatment(s). Patients were diagnosed based on the criteria of the 10th Revision of the International Classification of Diseases and Health Related Problems (ICD-10; World Health Organization [WHO], 1993). Cognition was measured with the Mini Mental Status Examination, MMSE (Folstein et al., 1975) and Informant Questionnaire on Cognitive Decline in the Elderly (IQCODE-16; Jorm and Jacomb, 1989). At the 1-year follow-up assessment patients were also diagnosed using Winblad's criteria for MCI (Winblad et al., 2004).

\section{Patient Selection}

Patients without dementia diagnoses according to ICD-10 criteria at inclusion and a T1W 3D/structural brain MRI were included in this study ( $n=95$, Figure 1$)$. Not all the patients could be included in the final analyses for a variety of reasons, as specified in Figure 1. Based on the 1-year follow-up diagnosis the patients were divided into one mild cognitive impairmentdementia (MCI-DEM) group including those diagnosed with either MCI $(n=21$; Winblad et al., 2004) or dementia $(n=8$, including: Alzheimer's disease $n=5$, unspecified dementia $n=3$ )
[World Health Organization (WHO)]. A matched cognitively stable (CS) group was selected from the remaining 51 patient based on age and gender $(n=40)$ and controlling for the balance in number of CS and MCI at each center of inclusion (Figure 1).

\section{Ethics Statement}

The participating patients and caregivers were given oral and written information, and they subsequently gave their written consent to participate. The PRODE study was approved by Regional Committee of Medical Research Ethics and Privacy and Data Protection Officer at Oslo University Hospital (approval number 2009/1774).

\section{MRI}

\section{MRI Acquisition}

All scans were optimized and harmonized across centers based on American College of Radiology (ACR) phantom and a healthy volunteer. This study is based on T1 3D brain MRI obtained with sagittal volumetric magnetization-prepared rapid gradient echo (3D MP-RAGE) images using the ADNI T1W volume protocol (Jack et al., 2008) in six PRODE centers with 1.5T and 3T MRI scanners (see Supplementary Table S1). Inter-site reliability for the six centers was estimated using the intra-class correlation coefficient (ICC) using two-way random effect model with absolute agreement (McGraw and Wong, 1996). ICC was estimated for the average CTH and total volume of subcortical structures (TSV) obtained from the Freesurfer parcellation and segmentation (see below). Reliability test was performed using SPSS software v. 22. Assessment showed acceptable results (Fleiss, 1986). ICC $(\mathrm{CTH})=0.88$ and ICC (TSV) $=0.98$. It has been shown that Freesurfer output measurements are consistent between 1.5T and 3T MRI (Han et al., 2006; Pfefferbaum et al., 
2012), thus the data across the field strengths was merged. CS group was matched on number of participants having 1.5 and $3 \mathrm{~T}$ scans in addition to the other criteria.

\section{Image Processing}

Cortical thickness and SV were used as main outcomes. The T1W 3D MRI brain images were processed using Freesurfer (v. 5.1), where regional cortical thicknesses and volumetric measures were estimated. The software is well documented and available for download online ${ }^{2}$. The processing steps included cortical reconstruction and segmentation of gray matter volumetric structures. This was followed by parcellation of the cerebral cortex into units based on gyral and sulcal structure (Destrieux et al., 2010). One hundred forty eight $\mathrm{CTH}$ measures (74 from each hemisphere) and 54 regional volumes were generated. All output was visually inspected for segmentation and parcellation quality before the analysis. Volumes of left and right white matter hypointensities, optic chiasm, right and left vessel, and left and right choroid plexus were excluded from further analysis. List of measurements included in the analysis is provided in Supplementary Table S2. The accuracy of the CTH and hippocampal volumes measurements derived by this technique has been validated by histological and manual measurements (Rosas et al., 2002; Sánchez-Benavides et al., 2010). All volumetric structures were normalized by the subject's intracranial volume using the residual approach (Jack et al., 1989). Brain structures in the left and right hemisphere may have different degree of atrophy, thus $\mathrm{CTH}$ and SV of the left and right hemisphere were treated separately (Dolcos et al., 2002). Workflow is represented in Figure 2 .

\section{Statistical Analysis}

$\mathrm{R}$ programming language ( $\mathrm{R}$ Core Team, 2016), version 3.3.0, was used to compare demographic and clinical data, and to create random-forest algorithm (RF) classification models. For group comparisons of demographic and clinical variables, the chi-squared test was used for categorical variables, and $t$-test or Mann-Whitney where appropriate for continuous variables. RF classification models were established using $\mathrm{R}$ packages "random forest" (Liaw and Wiener, 2002) and "caret" (Kuhn et al., 2016), ROC-curve and area under the ROC-curve (AUC) were estimated and plotted using "pROC" package (Robin et al., 2011). Regions important for the classification were correlated with clinical and demographic variables.

\section{Random Forest Algorithm and Performance Assessment}

Random forest method allows performing supervised classifications based on an ensemble of classification trees (Breiman, 2001). RF selects a bootstrapped subset of all observations - about $66 \%$ per tree and random subset of all predictors/features (here: $\mathrm{CTH}, \mathrm{SV}$ ) at each node of the tree. $\mathrm{RF}$ uses the majority vote of its trees terminal nodes to predict the class label of a new observation. Each tree casts a unit

${ }^{2}$ http://surfer.nmr.mgh.harvard.edu/ vote for the class. Thus, high numbers of decision trees are expected to increase reliability of the results. For each predictor a Gini index is estimated at each node. Overall importance of a predictor for the model is based on the summation of the decreases in the Gini index at each node (Breiman, 2001). The remaining $33 \%$ of the data, i.e., out-of-bag (OOB) data, is used to measure the RF performance. The classification error of the OOB observations is referred to as OOB error (Breiman, 1996). Kappa is another measure of performance demonstrating how close the RF classifications were to the actual classes, controlling for the accuracy of a random classifier as measured by the expected accuracy. Kappa is suggested to provide more reliable information regarding the classifier performance than actual accuracy in case of different class distribution in the dataset (Fleiss, 1971).

\section{RF Procedure}

Random forest method algorithm was used to discriminate between the MCI-DEM or MCI and CS groups at 1-year followup based on the CTH and SV measures separately and combined. In addition, demographic and clinical information was added to the models to test if performance could be further improved. Only the clinical information obtained at inclusion was used to assess if MCI-DEM statuses 1-year later could be predicted based on the earliest available clinical data and in the depressive state.

\section{PRODE Cohort Models \\ PRODE models}

Five thousand decision trees were used in the RF classification models. RF models were trained to discriminate between MCIDEM $(n=29)$ or MCI alone $(n=21)$ and corresponding CS patients. When discriminating between MCI vs. CS, the number of CS was reduced to 30 (from 40) matched on age and gender and scanner field strength, in order to keep balance in group class distribution. AUC, sensitivity/specificity, overall accuracy, and kappa, were used to assess performance of the models. Confidence intervals (CI) were estimated using bootstrapping $(n=100)$. The most relevant structures for prediction of MCIDEM or MCI were correlated with clinical variables related to depression in order to detect regions involved in both pathological processes.

\section{ADNI cohort models}

The previously established ADNI model (Lebedev et al., 2014) was implemented in the PRODE dataset to evaluate its ability to discriminate between MCI-DEM $(n=29)$ and CS group $(n=51)$. However, we did not expect the ADNI model to outperform the PRODE models because it was trained on a data derived from AD patients versus $\mathrm{HC}$, not LLD and not converting to MCI.

\section{RESULTS}

\section{Demographics}

Clinical and demographic variables in MCI-DEM and CS groups are provided in Table 1. MCI-DEM group had significantly lower MMSE scores at all three time points compared with 


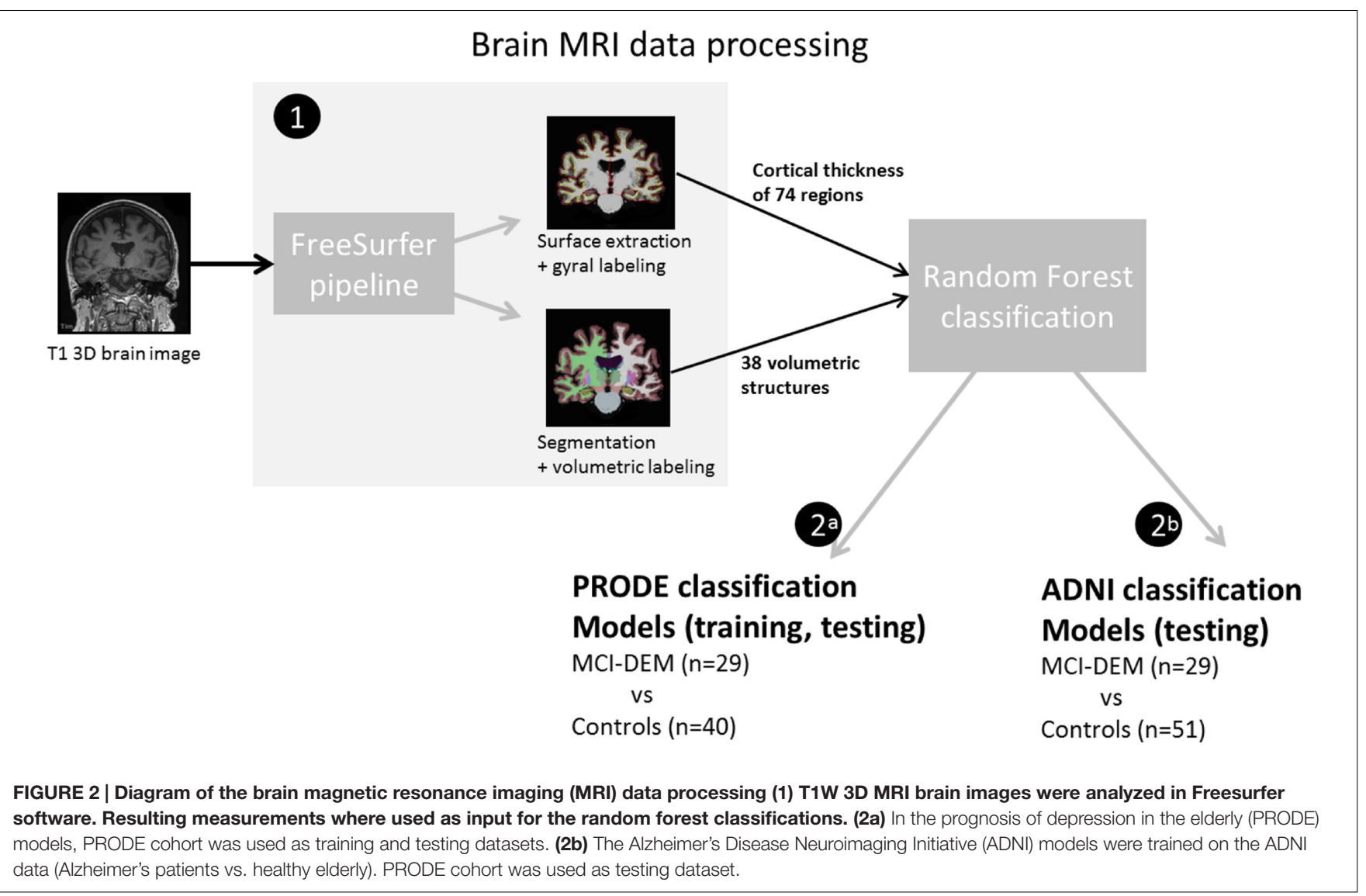

CS group. Other variables did not differ between the groups. Similarly, the MCI group alone did not differ from the matched CS patients in terms of age $(p=0.21)$ or gender $(p=0.92)$. Notably, the standard deviation of the MMSE scores increased in the MCI/dementia group between assessments "at discharge" and "follow-up," reflecting increased variance in their cognitive statuses.

\section{Prediction of $\mathrm{MCl}$ and Dementia in LLD PRODE (MCI-DEM vs. CS Group) Models}

The model using SV+CTH as input had the best performance in discrimination between MCI-DEM and CS groups. The model using only SV as input had the best performance in discrimination between MCI (excluding eight dementia patients) and CS groups. Details are provided in Table 2. The model using only CTH as input for the MCI vs. CS discrimination had $67 \%$ accuracy and sensitivity $<50 \%$. MMSE score at inclusion improved the models performance. Adding age, gender, or education to the model did not affect the results. When excluding patients with MADRS $<7$ and MMSE $<26$ at discharge $(n=4)$, accuracy changed slightly from 76 to $74 \%$ using SV as input.

\section{Brain Measurements Used in Prediction}

The most relevant measurements for the models were right ventral diencephalon (R-VD, mean decrease in Gini index $=8.26$ ), middle anterior corpus callosum (mid-anterior
$\mathrm{CC}$, mean decrease in Gini index $=2.06$ ) and right hippocampus (R-HC mean decrease in Gini index = 1.47). For all the PRODE models (Table 2), the same structures (R-VD, mid-anterior $\mathrm{CC}$, and $\mathrm{R}-\mathrm{HC}$ ) were the most important. When excluding participants from each center, the same structures remained the most important.

\section{Relationship between Ventral Diencephalon and Clinical and Demographic Measures}

Right ventral diencephalon, mid-anterior CC, and R-HC volumes were correlated with the number of depressive episodes (adjusting for age, gender, and MMSE). The number of depressive episodes had significant inverse association with R-VD $(p=0.02)$ and mid-anterior CC $(p=0.04)$ volumes. Next, total ventricular volume was added as a covariate to assess if structural changes co-occur with ventricle expansion as an indirect measure of a degenerative nature of the observed structural changes. Adjusting for total ventricular volume negated the effect of number of depressive episodes on R-VD volume $(p=0.12)$ and mid-anterior $\mathrm{CC}$ volume $(p=0.08)$. No association was found between the number of depressive episodes and R-HC volume. MMSE score was associated with R-HC volume $(p=0.03)$, but not with R-VD volume $(p=0.16)$ or mid-anterior CC volume $(p=0.18)$.

\section{ADNI (MCl-DEM vs. CS Group) Model}

The ADNI model trained on SV demonstrated better performance on separating MCI-DEM vs. CS in the PRODE 
TABLE 1 | Demographic and clinical characteristics of the MCI-DEM and cognitively stable (CS) groups.

\begin{tabular}{|c|c|c|c|c|}
\hline PRODE data set & MCI-DEM & CS & Test statistics & $p$-value* \\
\hline$N$ & 29 & 40 & - & - \\
\hline Age - mean (SD) & $78.1(7,3)$ & $76,4(5,8)$ & $t=-1.5$ & 0.31 \\
\hline Sex - n women (\%) & $22(75 \%)$ & $29(72 \%)$ & $x^{2}=0.03$ & 0.85 \\
\hline Education (total years) - mean (SD) & $8.9(2.79)$ & $10.1(2.61)$ & $t=-1.8$ & 0,07 \\
\hline Number of depressive episodes - (range) & $2.5(0-4)$ & $1.5(0-4)$ & $z=-1.06$ & 0.28 \\
\hline Age at depression onset - median (range) & $29.7(15-85)$ & $35.0(16-88)$ & $z=-1.02$ & 0.3 \\
\hline MADRS-1 - median (range) & $26(15-52)$ & $27(0-48)$ & $z=-0.66$ & 0.5 \\
\hline MADRS-2 & $9(0-31)$ & $7(0-30)$ & $z=-0.44$ & 0.65 \\
\hline MADRS-3 & $8(0-35)$ & $4.5(0-30)$ & $z=-0.43$ & 0.66 \\
\hline MMSE-1 - mean $(S D)$ & $24.6(3.0)$ & $26.4(1,8)$ & $t=-2.71$ & 0.005 \\
\hline MMSE-2 & $25(2.8)$ & $27(1.9)$ & $t=-5.42$ & 0.009 \\
\hline MMSE-3 & $23.8(5.6)$ & $28(1.7)$ & $t=-5.90$ & $<0.001$ \\
\hline
\end{tabular}

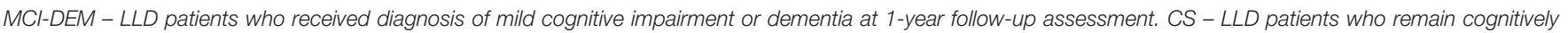
stable at 1-year follow-up assessment.

*t-test, Mann-Whitney $U$ test or chi-square test as appropriate.

MADRS, Montgomery-Asberg Depression Rating Scale; MMSE, Mini Mental State Examination.

MADRS/MMSE - 1 - at inclusion, 2- at discharge, 3- after 1 year follow-up period.

dataset (accuracy $=67.0 \%$ ) than the ADNI model trained on $\mathrm{CTH}+\mathrm{SV}$ (accuracy $=57.5 \%$; Table 2 ).

\section{DISCUSSION}

In this study, we have demonstrated that LLD patients who were diagnosed with MCI or dementia 1 year later can be discriminated from cognitively stable LLD patients using structural brain measurements with $76 \%$ accuracy. The best model predicted MCI status alone using SV and MMSE scores with accuracy/sensitivity/specificity of $89 \% / 85 \% / 90 \%$.

To our knowledge this study is the first to build classification models based on structural MRI measures to predict development of MCI or dementia in LLD patients, and to assess performance of a model trained for $\mathrm{AD}-\mathrm{HC}$ discrimination (ADNI model) on a LLD dataset (PRODE cohort). We used all brain parenchyma volumes derived from the Freesurfer analysis, and showed that volumes of R-VD, mid-anterior CC, and R-HC were the most important for discrimination between MCI-DEM and CS group.

We have found only one study using a classification approach and MRI data to predict MCI diagnosis, but in non-depressed elderly and based on arterial spin labeling. This study reported that perfusion in a region of interest in the posterior cingular cortex could distinguish those developing MCI from a CS group with an AUC of 66\% (Xekardaki et al., 2015).

\section{Brain Regions Important for Prediction of $\mathrm{MCl}$ and Dementia Diagnosis}

The most important regions for predicting a diagnosis of MCI or dementia after 1-year follow-up were volumes of R-VD, midanterior CC, and R-HC, respectively.

Right ventral diencephalon was demonstrated to be the most relevant structure. The ventral diencephalon in Freesurfer includes several structures: hypothalamus with mammillary body, subthalamic, lateral geniculate, medial geniculate and red nuclei, substantia nigra and surrounding white matter. Some of these structures, i.e., substantia nigra and red nuclei, are not locate to the diencephalon but mesencephalon according to standard anatomical nomenclature. The hypothalamus is known to be strongly involved in depression. As a part of hypothalamic-pituitary-adrenal (HPA) axis, it is crucial for emotional behavior and stress response. There are numerous studies showing dysregulation of the HPA axis in depression, but also in aging and neurodegeneration (Sapolsky et al., 1986; Sapolsky, 2000; Varghese and Brown, 2001; Du and Pang, 2015). It has also been proposed that HPA-axis dysfunction is central to the development of AD (Ishii and Iadecola, 2015). Indeed, hypothalamic dysfunction can explain the overlap in symptoms between depression and $\mathrm{AD}$ (mood, appetite, sleep, memory, autonomic). Consistent with our finding, several previous imaging studies have shown structural and functional abnormalities in the hypothalamus in MCI, preclinical AD, and AD compared with control groups (Callen et al., 2001; Nestor et al., 2003; Cross et al., 2013; Brueggen et al., 2015). For instance, Hall et al. (2008) have demonstrated reduced basal forebrain and hypothalamus volumes in preclinical $\mathrm{AD}$, and interestingly the combination of reduced forebrain and hippocampal volumes was associated with more rapid cognitive decline. However, to the best of our knowledge the current study is the first study to assess the role of the entire ventral diencephalon in the context of neurodegeneration. There are two previous studies examining the ventral diencephalon (segmented in Freesurfer) in relation to mood disorders. The first showed that bilateral ventral diencephalon volume obtained from Freesurfer was one of three top-ranked endophenotypes of major depressive disorder in an analysis of a high-dimensional set of over 11,000 traits (Glahn et al., 2012). The other study demonstrated that volume of the ventral diencephalon discriminated patients with major depressive disorder from those with bipolar depression as well as controls (Sacchet et al., 2015). Taken together, these 
TABLE 2 | Performance of the random forest classification models based on baseline structural magnetic resonance imaging (MRI) measurements with and without Mini-Mental State Examination scores.

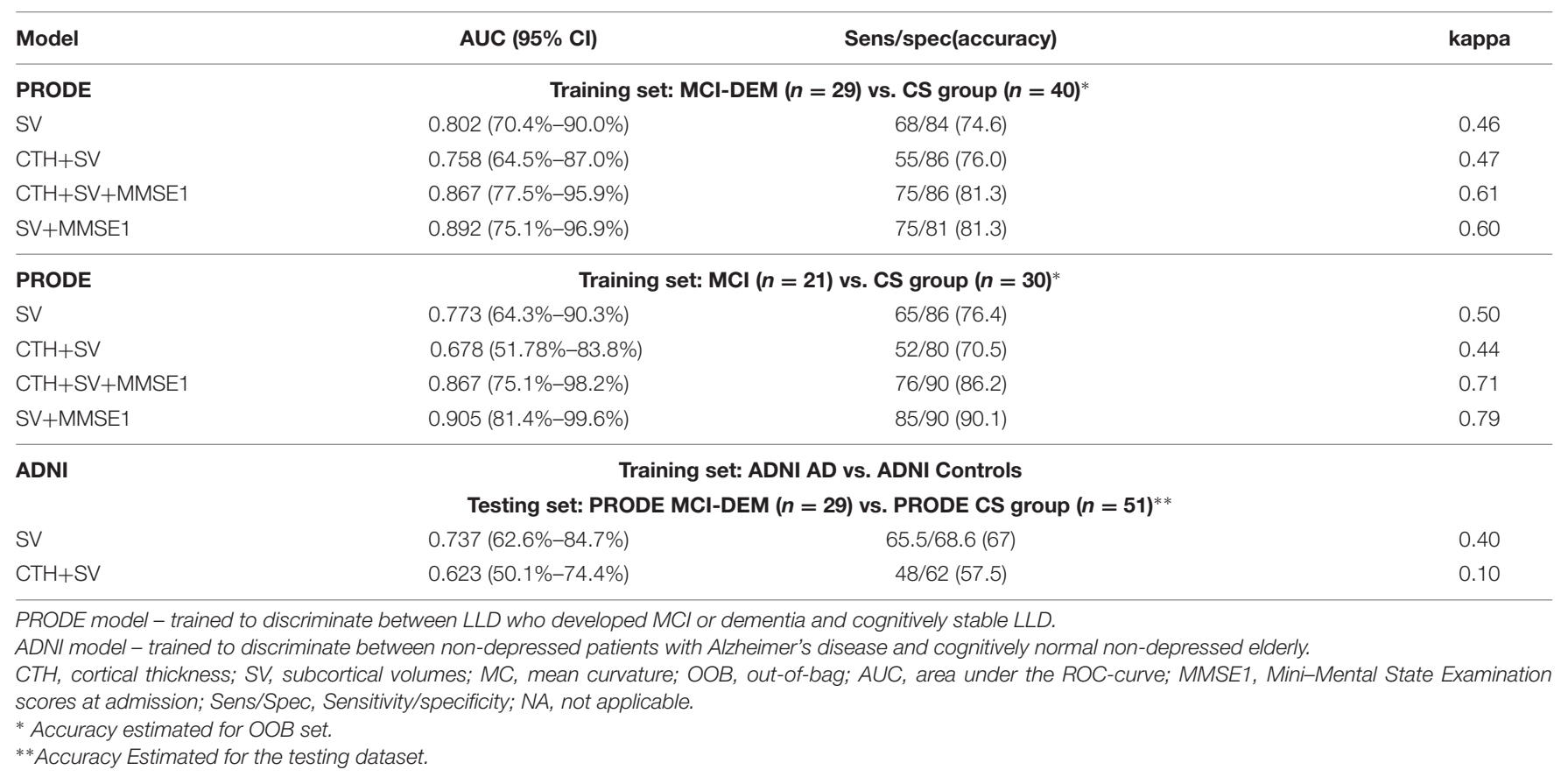

findings suggest that neurodegeneration in ventral diencephalon including the hypothalamus might be a link between depression and cognitive impairment.

After R-VD, CC (mid-anterior CC) and hippocampal (R$\mathrm{HC}$ ) volumes were the most relevant structures for predicting MCI-DEM. HPA-axis dysregulation is associated with elevated cortisol levels (O'Brien, 1996; Du and Pang, 2015) which is hypothesized to cause reduced CC and hippocampal volumes due to neurotoxic effects (Bao et al., 2008; Liu et al., 2016). The importance of CC volume for predicting MCI-DEM suggests presence of inter-hemispheric disconnection already in the early stages of the neurodegenerative process. Previously, it has been shown that anterior, middle and posterior portions of the CC have less volume in $\mathrm{AD}$ compared to controls, but only the middle part was smaller in amnestic MCI compared with controls (Qiu et al., 2016). Moreover, decreased volume of mid-anterior portion of $\mathrm{CC}$ has been linked to the memory loss in $\mathrm{MCI}$ and $\mathrm{AD}$ (Qiu et al., 2016). The present findings support a connection between R-VD, R-HC, and CC pathology in the development of MCI and dementia in LLD, which may be linked to HPAaxis dysregulation. A previous study examining only CC volume reported that structural changes in CC predicted MCI-to-AD conversion after 2.5 years on average (Lee et al., 2016), similar to the current study where a whole brain approach was used and conversion was to $\mathrm{MCI} / \mathrm{dementia}$. Hippocampal abnormalities are one of the most replicable findings in both depression and $\mathrm{AD}$ (Kempton et al., 2011; Sabuncu et al., 2011). Hippocampus has large bidirectional connection with the mammillary bodies of the hypothalamus which also might explain the concordant changes in these two structures. The limbic-diencephalic pathways, including the mammillothalamic tract and the mammillary bodies per se are crucial for episodic memory (Vann and Nelson, 2015; Aggleton et al., 2016). Hypometabolism in the mammillary bodies has been shown in both MCI and AD (Nestor et al., 2003). Limbic-diencephalic pathway dysregulation has been shown in the earliest stages of AD (Acosta-Cabronero and Nestor, 2014). Indeed, limbic regions, which are crucial for emotion processing, are also crucial for episodic memory. Thus, abnormal limbicdiencephalic interaction may be a core feature of in MCI-DEM development in LDD.

Of note, only brain structures from the right hemisphere were important for the classifications. This supports the right hemiaging model, proposing that the right hemisphere shows greater age-related decline than the left hemisphere (Dolcos et al., 2002). However, this model is based on behavioral data rather than neuroimaging and the evidence has been controversial (Daselaar and Cabeza, 2005).

Interestingly, the number of depressive episodes had significant inverse association with the R-VD and mid-anterior CC volumes. Previously, it was reported that the number of depressive episodes is associated with reduced volume of the dentate gyrus in patients with major depressive disorder (Treadway et al., 2015). It is not known whether reductions of $\mathrm{R}-\mathrm{VD}$ and mid-anterior CC are developmental phenomena, which leads to increase of the number of depressive episodes or degenerative consequences of a larger number of depressive episodes. However, including total ventricular volume to the regression model negated the effect of the number of depressive episodes on the R-VD volume and reduced on mid-anterior $\mathrm{CC}$, providing indirect evidence for the degenerative nature of R-VD and mid-anterior CC reduction. Future studies using longitudinal imaging data could uncover the causality. 


\section{SV+CTH+MMSE Models}

MCI-DEM had significantly lower MMSE scores at all three time points compared to the CS group. Regardless of whether cognitive assessment in depressed patients is confounded by depression per se, including MMSE scores at admission improved the model's prediction of MCI and dementia. Along with a recent study of Heser et al. (2016), the present results suggest that even during the depressive episode cognitive impairment require clinical attention as a possible sign of incipient dementia. Given the highly significant difference in MMSE scores at all three time points between the MCI/DEM and CS group, the probability that there was a true difference in MMSE scores between MCI-DEM and CS groups at all time points is very high, suggesting that MMSE in an appropriate additional predictor of MCI/AD even in LLD groups.

There was no correlation between MMSE scores R-VD and mid-anterior CC volumes suggesting that MMSE scores did not bias classification results. The absence of correlation between MMSE and the two main predictors suggested that MMSE improved classification models performance by explaining additional factor variance.

\section{Predicting $\mathrm{MCl}-\mathrm{DEM}$ vs. $\mathrm{MCl}$}

The SV model trained on MCI vs. CS demonstrated better performance than the model trained on the mixed sample of MCI-DEM dementia vs. CS. The reasons might be a more heterogeneous pattern of structural brain changes across dementias in the MCI/DEM combined group and presence of larger variability in the stages of pathological process. On the other hand, the CTH+SV model performed better on the mixed MCI+DEM group compared with MCI only. One explanation might be that CTH get altered in the later stages and/or more severe cases of cognitive impairment in LLD and that relevance of the structural brain measurement as a biomarker therefore depends on the stage of the disease.

\section{Predicting MCI-DEM Conversion in LLD Using the ADNI Model}

MCI-DEM status was discriminated from the CS group with $67 \%$ accuracy using the ADNI model. In other words, patients in the MCI-DEM group were more likely to be classified as cases than those in CS group based on their baseline brain MRI.

Interestingly, the ADNI model trained only on SV had much higher accuracy compared with the one trained on CTH+SV. Taking into account that the most relevant structures for MCICS discrimination (PRODE models) were subcortical structures and that the model combining SV with $\mathrm{CTH}$ had higher accuracy in MCI-DEM than in MCI alone, the present results may suggest that in LLD patients, who will develop MCI or dementia, neurodegeneration appears to start in subcortical structures and spread up to the neocortex in later stages. In agreement with our findings it has been shown that atrophy in basal forebrain and hypothalamus but not neocortex, precedes clinical symptoms of AD by 4.5-5 years (Brueggen et al., 2015). Several earlier studies have shown hypometabolism restricted to hippocampus and parahippocampal gyrus in $\mathrm{MCI}$, whereas $\mathrm{AD}$ had additional temporal neocortical hypometabolism (De Santi et al., 2001; Nestor et al., 2003). Taken together, the current results suggest that classification models for prediction of $\mathrm{MCI} /$ preclinical AD should focus on subcortical structures rather than the neocortex.

\section{Limitations}

One of the limitations of the present study is that the time interval between MRI assessment and MCI diagnosis was relatively short, thus it is possible that some CS patients could be diagnosed with $\mathrm{MCI} /$ dementia at a later time point. Excluding LLD participants with the lowest MADRS and MMSE scores at discharge $(n=4)$ did not alter the results notably (no substantial drop in accuracy) which indicate that the possibility that some patients could receive MCI diagnosis earlier did not bias the results strongly. Another issue is that MMSE might not be sensitive enough to detect MCI. In any case, there are no cognitive screening instruments validated for use in a depressed elderly population according to our knowledge. All the models including only brain measurements as predictors had very good specificity and sufficient sensitivity. One of the reasons for this might be the relatively small sample size. In future studies classification models should be trained on larger samples after longer follow-up periods. Inclusion of MRI scans obtained from scanners with different magnetic field strengths might be considered a limitation; however, it was shown that Freesurfer output measurements are consistent between $1.5 \mathrm{~T}$ and $3 \mathrm{~T} \mathrm{MRI}$, which was confirmed in our reliability analysis. We have shown that ICC is high across the centers. We also balanced the number of participants from each center and verified the results excluding each center, in order to assess reliability of the results which were consistent.

\section{Possible Practical Implementation}

Our results suggest that LLD with smaller volumes of the R-VD, mid-anterior CC, and R-HC and lower MMSE scores at inclusion have a higher probability of receiving a diagnosis of $\mathrm{MCI}$ or dementia the following year. These measurements can provide clinicians with novel evidence for an expected trajectory of cognitive functioning in LLD and help to define a target group for interventions against cognitive decline (Bredesen, 2014).

Future mechanistic studies should verify processes underlying diencephalic neurodegeneration in LLD patients.

\section{AUTHOR CONTRIBUTIONS}

AL: Study design, data analyses, interpretation of the results, manuscript writing. EW and DA: Study design, interpretation of the results. TB and KE: Study design, data collection, interpretation of the results. MB: Data collection, interpretation of the results. GS and AH: Data collection, study design, interpretation of the results. All authors participated in manuscript revision and final approval. 


\section{FUNDING}

The PRODE-study was funded by unrestricted grants from the South-Eastern Norway Regional Health Authority (grant number: 2010088) and Innlandet Hospital Trust (grant number: 150201). The PRODE-study also received some funding from: Solveig and Johan P. Sommer's Foundation (2010-3) for Psychiatric Research and The Legacy of Josef and Haldis Andresen. AL received a Ph.D. grant from Swedish Brain Power foundation and the Strategic Research Program in Neuroscience at Karolinska Institutet. TB received a Ph.D. grant from the South-Eastern Norway Regional Health Authority and Innlandet Hospital Trust. The sponsors had no role in the study design, data collection, data analysis, data interpretation or writing of the report.

\section{ADNI}

Data collection and sharing for this project was funded by the Alzheimer's Disease Neuroimaging Initiative (ADNI) (National Institutes of Health Grant U01 AG024904) and DOD ADNI (Department of Defense award number W81XWH-12-2-0012). ADNI is funded by the National Institute on Aging, the National Institute of Biomedical Imaging and Bioengineering, and through generous contributions from the following: AbbVie, Alzheimer's Association; Alzheimer's Drug Discovery Foundation; Araclon Biotech; BioClinica, Inc.; Biogen; BristolMyers Squibb Company; CereSpir, Inc.; Cogstate; Eisai Inc.; Elan Pharmaceuticals, Inc.; Eli Lilly and Company; EuroImmun; F. Hoffmann-La Roche Ltd. and its affiliated company Genentech, Inc.; Fujirebio; GE Healthcare; IXICO Ltd.; Janssen Alzheimer Immunotherapy Research \& Development, LLC.; Johnson

\section{REFERENCES}

Acosta-Cabronero, J., and Nestor, P. J. (2014). Diffusion tensor imaging in Alzheimer's disease: insights into the limbic-diencephalic network and methodological considerations. Front. Aging Neurosci. 6:266. doi: 10.3389/fnagi. 2014.00266

Aggleton, J. P., Pralus, A., Nelson, A. J. D., and Hornberger, M. (2016). Thalamic pathology and memory loss in early Alzheimer's disease: moving the focus from the medial temporal lobe to Papez circuit. Brain 139, 1877-1890. doi: 10.1093/brain/aww083

American Psychiatric Association (2013). DSM-5: Diagnostic and Statistical Manual of Mental Disorders. Arlington, TX: American Psychiatric Association.

Aziz, R., and Steffens, D. (2013). What are the causes of late-life depression? Psychiatr. Clin. North Am. 36, 497-516. doi: 10.1016/j.psc.2013.08.001

Bao, A. M., Meynen, G., and Swaab, D. F. (2008). The stress system in depression and neurodegeneration: focus on the human hypothalamus. Brain Res. Rev. 57, 531-553. doi: 10.1016/j.brainresrev.2007.04.005

Borza, T., Engedal, K., Bergh, S., Benth, J. Š, and Selbæk, G. (2015). The course of depression in late life as measured by the montgomery and asberg depression rating scale in an observational study of hospitalized patients. BMC Psychiatry 15:191. doi: 10.1186/s12888-015-0577-8

Bredesen, D. E. (2014). Reversal of cognitive decline: a novel therapeutic program. Aging (Albany N.Y.) 6, 707-717.

Breiman, L. (1996). Out-of-Bag Estimation. Technical report, Statistics Department. Berkeley CA: University of California Berkeley.

Breiman, L. (2001). Random forests. Mach. Learn. 45, 5-32. doi: 10.1023/A: 1010933404324
\& Johnson Pharmaceutical Research \& Development LLC.; Lumosity; Lundbeck; Merck \& Co., Inc.; Meso Scale Diagnostics, LLC.; NeuroRx Research; Neurotrack Technologies; Novartis Pharmaceuticals Corporation; Pfizer Inc.; Piramal Imaging; Servier; Takeda Pharmaceutical Company; and Transition Therapeutics. The Canadian Institutes of Health Research is providing funds to support ADNI clinical sites in Canada. Private sector contributions are facilitated by the Foundation for the National Institutes of Health (www.fnih.org). The grantee organization is the Northern California Institute for Research and Education, and the study is coordinated by the Alzheimer's Therapeutic Research Institute at the University of Southern California. ADNI data are disseminated by the Laboratory for Neuro Imaging at the University of Southern California.

\section{ACKNOWLEDGMENTS}

\section{PRODE}

The authors thank MRI technologist Ingeborg Nakken, Norwegian National Advisory Unit for functional MRI, St. Olav's University Hospital for MRI scan harmonization, image quality assurance and anonymization of scans, Dr. Alexander Lebedev for providing ADNI classification models.

\section{SUPPLEMENTARY MATERIAL}

The Supplementary Material for this article can be found online at: http://journal.frontiersin.org/article/10.3389/fnagi. 2017.00013/full\#supplementary-material

Brueggen, K., Dyrba, M., Barkhof, F., Hausner, L., Filippi, M., Nestor, P. J., et al. (2015). Basal forebrain and hippocampus as predictors of conversion to alzheimer's disease in patients with mild cognitive impairment - a multicenter DTI and volumetry study. J. Alzheimers Dis. 48, 197-204. doi: 10.3233/JAD150063

Byers, A. L., and Yaffe, K. (2014). Depression and dementias among military veterans. Alzheimers Dement. 10, S166-S173. doi: 10.1016/j.jalz.2014. 04.007

Callen, D. J., Black, S. E., Gao, F., Caldwell, C. B., and Szalai, J. P. (2001). Beyond the hippocampus: MRI volumetry confirms widespread limbic atrophy in AD. Neurology 57, 1669-1674. doi: 10.1212/WNL.57.9.1669

Cooper, C., Sommerlad, A., Lyketsos, C. G., and Livingston, G. (2015). Modifiable predictors of dementia in mild cognitive impairment: a systematic review and meta-analysis. Am. J. Psychiatry 172, 323-334. doi: 10.1176/appi.ajp.2014. 14070878

Cross, D. J., Anzai, Y., Petrie, E. C., Martin, N., Richards, T. L., Maravilla, K. R., et al. (2013). Loss of olfactory tract integrity affects cortical metabolism in the brain and olfactory regions in aging and mild cognitive impairment. J. Nucl. Med. 54, 1278-1284. doi: 10.2967/jnumed.112.116558

Daselaar, S. M., and Cabeza, R. (2005). "Age-Related Changes in Hemispheric Organization," in Cognitive Neuroscience of Aging: Linking Cognitive and Cerebral Aging, eds R. Cabeza, L. Nyberg, and D. C. Park (New York, NY: Oxford University Press), 325-353.

De Santi, S., De Leon, M. J., Rusinek, H., Convit, A., Tarshish, C. Y., Roche, A., et al. (2001). Hippocampal formation glucose metabolism and volume losses in MCI and AD. Neurobiol. Aging 22, 529-539. doi: 10.1016/S0197-4580(01) 00230-5 
Destrieux, C., Fischl, B., Dale, A., and Halgren, E. (2010). Automatic parcellation of human cortical gyri and sulci using standard anatomical nomenclature. Neuroimage 34, 1-15. doi: 10.1016/j.neuroimage.2010.06.010

Dickerson, B. C., and Wolk, D. A. (2013). Biomarker-based prediction of progression in $\mathrm{MCI}$ : comparison of $\mathrm{AD}$ signature and hippocampal volume with spinal fluid amyloid- $\beta$ and tau. Front. Aging Neurosci. 5:55. doi: 10.3389/ fnagi.2013.00055

Diniz, B. S., Butters, M. A., Albert, S. M., Dew, M. A., and Reynolds, C. F. (2013). Late-life depression and risk of vascular dementia and Alzheimer's disease: systematic review and meta-analysis of community-based cohort studies. $\mathrm{Br}$. J. Psychiatry 202, 329-335. doi: 10.1192/bjp.bp.112.118307

Dolcos, F., Ricea, H. J., and Cabeza, R. (2002). Hemispheric asymmetry and aging?: right hemisphere decline or asymmetry reduction Hemispheric asymmetry and aging?: right hemisphere decline or asymmetry reduction. Neurosci. Biobehav. Rev. 7634, 819-825. doi: 10.1016/S0149-7634(02)00068-4

Du, X., and Pang, T. Y. (2015). Is dysregulation of the HPA-axis a core pathophysiology mediating co-morbid depression in neurodegenerative diseases? Front. Psychiatry 6:32. doi: 10.3389/fpsyt.2015.00032

du Jardin, K. G., Müller, H. K., Sanchez, C., Wegener, G., and Elfving, B. (2016). A single dose of vortioxetine, but not ketamine or fluoxetine, increases plasticity-related gene expression in the rat frontal cortex. Eur. J. Pharmacol. 786, 29-35. doi: 10.1016/j.ejphar.2016.05.029

Fleiss, J. L. (1971). Measuring nominal scale agreement among many raters 1. Psychol. Bull. 76, 378-382. doi: 10.1037/h0031619

Fleiss, J. L. (1986). Analysis of data from multiclinic trials. Control Clin. Trials 7, 267-275. doi: 10.1016/0197-2456(86)90034-6

Folstein, M. F., Folstein, S. E., and McHugh, P. R. (1975). "Mini-mental state". A practical method for grading the cognitive state of patients for the clinician. J. Psychiatr. Res 12, 189-198. doi: 10.1016/0022-3956(75)90026-6

García-Fuster, M. J., and García-Sevilla, J. A. (2016). Effects of anti-depressant treatments on FADD and p-FADD protein in rat brain cortex: enhanced anti-apoptotic p-FADD/FADD ratio after chronic desipramine and fluoxetine administration. Psychopharmacology (Berl) 233, 2955-2971. doi: 10.1007/ s00213-016-4342-6

Glahn, D. C., Curran, J. E., Winkler, A. M., Carless, M. A., Kent, J. W., Charlesworth, J. C., et al. (2012). High dimensional endophenotype ranking in the search for major depression risk genes. Biol. Psychiatry 71, 6-14. doi: 10.1016/j.biopsych.2011.08.022

Hall, A. M., Moore, R. Y., Lopez, O. L., Kuller, L., and Becker, J. T. (2008). Basal forebrain atrophy is a presymptomatic marker for Alzheimer's disease. Alzheimers Dement. 4, 271-279. doi: 10.1016/j.jalz.2008.04.005

Han, X., Jovicich, J., Salat, D., van der Kouwe, A., Quinn, B., Czanner, S., et al. (2006). Reliability of MRI-derived measurements of human cerebral cortical thickness: the effects of field strength, scanner upgrade and manufacturer. Neuroimage 32, 180-194. doi: 10.1016/j.neuroimage.2006. 02.051

Heser, K., Bleckwenn, M., Wiese, B., Mamone, S., Riedel-Heller, S. G., Stein, J., et al. (2016). Late-life depressive symptoms and lifetime history of major depression: cognitive deficits are largely due to incipient dementia rather than depression. $J$ Alzheimers Dis. 54, 185-199. doi: 10.3233/JAD- 160209

Ishii, M., and Iadecola, C. (2015). Metabolic and non-cognitive manifestations of alzheimers disease: the hypothalamus as both culprit and target of pathology. Cell Metab. 22, 761-776. doi: 10.1016/j.cmet.2015.08.016

Jack, C. R., Bernstein, M. A., Fox, N. C., Thompson, P., Alexander, G., Harvey, D., et al. (2008). The Alzheimers disease neuroimaging initiative (ADNI): MRI methods. J. Magn. Reson. Imaging 27, 685-691. doi: 10.1002/jmri. 21049

Jack, R., Twomey, K., Sharbrough, F. W., Petersen, C., and Cascino, D. (1989). Anterior temporal lobes and hippocampal formations: normative volumetric measurements from MR images in young adults. Radiology 172, 549-554. doi: 10.1148/radiology.172.2.2748838

Jorm, A. F., and Jacomb, P. A. (1989). The informant questionnaire on cognitive decline in the elderly (IQCODE): socio-demographic correlates, reliability, validity and some norms. Psychol. Med. 19, 1015-1022. doi: 10.1017/ S0033291700005742

Kempton, M. J., Salvador, Z., Munafò, M. R., Geddes, J. R., Simmons, A., Frangou, S., et al. (2011). Structural neuroimaging studies in major depressive disorder. Meta-analysis and comparison with bipolar disorder.
Arch. Gen. Psychiatry 68, 675-690. doi: 10.1001/archgenpsychiatry. 2011.60

Koutsouleris, N., Davatzikos, C., Borgwardt, S., Gaser, C., Bottlender, R., Frodl, T., et al. (2014). Accelerated brain aging in schizophrenia and beyond: a neuroanatomical marker of psychiatric disorders. Schizophr. Bull. 40, 11401153. doi: 10.1093/schbul/sbt142

Kuhn, M., Wing, J., Weston, S., Williams, A., Engelhardt, A., Mayer, Z., et al. (2016). caret: Classification and Regression Training. R Package Version 6.0-70. Available at: https://CRAN.R-project.org/package $=$ caret

Lebedev, A. V., Westman, E., Van Westen, G. J. P., Kramberger, M. G., Lundervold, A., Aarsland, D., et al. (2014). Random Forest ensembles for detection and prediction of Alzheimer's disease with a good between-cohort robustness. Neuroimage Clin. 6, 115-125. doi: 10.1016/j.nicl.2014.08.023

Lebedeva, A., Borza, T., Håberg, A. K., Idland, A.-V., Dalaker, T. O., Aarsland, D., et al. (2015). Neuroanatomical correlates of late-life depression and associated cognitive changes. Neurobiol. Aging 36, 3090-3099. doi: 10.1016/ j.neurobiolaging.2015.04.020

Lee, S. H., Bachman, A. H., Yu, D., Lim, J., and Ardekani, B. A. (2016). Predicting progression from mild cognitive impairment to Alzheimer's disease using longitudinal callosal atrophy. Alzheimers Dement (Amst.)2, 1-7. doi: 10.1016/ j.dadm.2016.01.003

Liaw, A., and Wiener, M. (2002). Classification and regression by randomForest. $R$ News 2, 18-22.

Liu, X., Watanabe, K., Kakeda, S., Yoshimura, R., Abe, O., Ide, S., et al. (2016). Relationship between white matter integrity and serum cortisol levels in drugnaive patients with major depressive disorder: diffusion tensor imaging study using tract-based spatial statistics. Br. J. Psychiatry 208, 585-590. doi: 10.1192/ bjp.bp.114.155689

McGraw, K. O., and Wong, S. P. (1996). Forming inferences about some intraclass correlation coefficients. Psychol. Methods 1, 30-46. doi: 10.1037/1082-989X.1.1. 30

McKinney, B., and Sibille, E. (2013). The age-by-disease interaction hypothesis of late-life depression. Am. J. Geriatr. Psychiatry 21, 1-19. doi: 10.1016/j.jagp.2013. 01.053

Mourao, R. J., Mansur, G., Malloy-Diniz, L. F., Castro Costa, E., and Diniz, B. S. (2015). Depressive symptoms increase the risk of progression to dementia in subjects with mild cognitive impairment: systematic review and meta-analysis. Int. J. Geriatr. Psychiatry 31, 905-911. doi: 10.1002/gps.4406

Nestor, P. J., Fryer, T. D., Smielewski, P., and Hodges, J. R. (2003). Limbic hypometabolism in Alzheimer's disease and mild cognitive impairment. Ann. Neurol. 54, 343-351. doi: 10.1002/ana.10669

Ngandu, T., Lehtisalo, J., Solomon, A., Levälahti, E., Ahtiluoto, S., Antikainen, R., et al. (2015). A 2 year multidomain intervention of diet, exercise, cognitive training, and vascular risk monitoring versus control to prevent cognitive decline in at-risk elderly people (FINGER): a randomised controlled trial. Lancet 385, 2255-2263. doi: 10.1016/S0140-6736(15)60461-5

O'Brien, J. T. (1996). Clinical and magnetic resonance imaging correlates of hypothalamic-pituitary-adrenal axis function in depression and Alzheimer's disease. Br. J. Psychiatry 168, 679-687. doi: 10.1192/bjp.168.6.679

Pfefferbaum, A., Rohlfing, T., Rosenbloom, M. J., and Sullivan, E. V. (2012). Combining atlas-based parcellation of regional brain data acquired across scanners at $1.5 \mathrm{~T}$ and $3.0 \mathrm{~T}$ field strengths. Neuroimage 60, 940-951. doi: 10.1016/j.neuroimage.2012.01.092

Qiu, Y., Liu, S., Hilal, S., Loke, Y. M., Ikram, M. K., Xu, X., et al. (2016). Interhemispheric functional dysconnectivity mediates the association of corpus callosum degeneration with memory impairment in AD and amnestic MCI. Sci. Rep. 6:32573. doi: 10.1038/srep32573

R Core Team (2016). R: A Language and Environment for Statistical Computing. Vienna: R Foundation for Statistical Computing.

Robin, X., Turck, N., Hainard, A., Tiberti, N., Lisacek, F., Sanchez, J.-C., et al. (2011). pROC: an open-source package for R and S+ to analyze and compare ROC curves. BMC Bioinformatics 12:77. doi: 10.1186/1471-2105-12-77

Rosas, H., Liu, A., Hersch, S., and Glessner, M. (2002). Regional and progressive thinning of the cortical ribbon in Huntington's disease. Neurology 58, 695-701. doi: 10.1212/WNL.58.5.695

Sabuncu, M. R., Desikan, R. S., Sepulcre, J., Yeo, B. T., Liu, H., Schmansky, N. J., et al. (2011). The Dynamics of cortical and hippocampal atrophy in Alzheimer disease. Arch. Neurol. 68, 1040-1048. doi: 10.1001/archneurol.2011.167 
Sacchet, M. D., Livermore, E. E., Iglesias, J. E., Glover, G. H., and Gotlib, I. H. (2015). Subcortical volumes differentiate major depressive disorder, bipolar disorder, and remitted major depressive disorder. J. Psychiatr. Res. 68, 91-98. doi: 10.1016/j.jpsychires.2015.06.002

Sánchez-Benavides, G., Gómez-Ansón, B., Sainz, A., Vives, Y., Delfino, M., and Peña-Casanova, J. (2010). Manual validation of FreeSurfer's automated hippocampal segmentation in normal aging, mild cognitive impairment, and Alzheimer disease subjects. Psychiatry Res. 181, 219-225. doi: 10.1016/j. pscychresns.2009.10.011

Sapolsky, R. M. (2000). Glucocorticoids and hippocampal atrophy in neuropsychiatric disorders. Arch. Gen. Psychiatry 57, 925-935. doi: 10. 1001/archpsyc.57.10.925

Sapolsky, R. M., Krey, L. C., and McEwen, B. S. (1986). The neuroendocrinology of stress and aging: the glucocorticoid cascade hypothesis. Endocr. Rev. 7, 284-301. doi: 10.1210/edrv-7-3-284

Simon, N. M., Smoller, J. W., McNamara, K. L., Maser, R. S., Zalta, A. K., Pollack, M. H., et al. (2006). Telomere shortening and mood disorders: preliminary support for a chronic stress model of accelerated aging. Biol. Psychiatry 60, 432-435. doi: 10.1016/j.biopsych.2006.02.004

Steffens, D. C., McQuoid, D. R., and Potter, G. G. (2009). Outcomes of older cognitively impaired individuals with current and past depression in the NCODE study. J. Geriatr. Psychiatry Neurol. 22, 52-61. doi: 10.1177/ 0891988708328213

Steffens, D. C., Otey, E., Alexopoulos, G. S., Butters, M. A., Cuthbert, B., Ganguli, M., et al. (2006). Perspectives on depression, mild cognitive impairment, and cognitive decline. Arch. Gen. Psychiatry 63, 130-138. doi: 10.1001/archpsyc.63.2.130

Treadway, M. T., Waskom, M. L., Dillon, D. G., Holmes, A. J., Park, M. T. M., Chakravarty, M. M., et al. (2015). Illness progression, recent stress, and morphometry of hippocampal subfields and medial prefrontal cortex in major depression. Biol. Psychiatry 77, 285-294. doi: 10.1016/j.biopsych.2014.06.018
Vann, S. D., and Nelson, A. J. D. (2015). The mammillary bodies and memory: more than a hippocampal relay. Prog. Brain Res. 219, 163-185. doi: 10.1016/bs. pbr.2015.03.006

Varghese, F. P., and Brown, E. S. (2001). The hypothalamic-pituitary-adrenal axis in major depressive disorder: a brief primer for primary care physicians. Prim. Care Companion J. Clin. Psychiatry 3, 151-155. doi: 10.4088/PCC.v03n 0401

Winblad, B., Palmer, K., Kivipelto, M., Jelic, V., Fratiglioni, L., Wahlund, L.O., et al. (2004). Mild cognitive impairment-beyond controversies, towards a consensus: report of the international working group on mild cognitive impairment. J. Intern. Med. 256, 240-246. doi: 10.1111/j.1365-2796.2004. 01380.x

World Health Organization [WHO] (1993). The ICD-10 Classification of Mental and Behavioural Disorders. Geneva: World Health Organization, $1-267$.

Xekardaki, A., Rodriguez, C., Montandon, M.-L., Toma, S., Tombeur, E., Herrmann, F. R., et al. (2015). Arterial spin labeling may contribute to the prediction of cognitive deterioration in healthy elderly individuals. Radiology 274, 490-499. doi: 10.1148/radiol.14140680

Conflict of Interest Statement: The authors declare that the research was conducted in the absence of any commercial or financial relationships that could be construed as a potential conflict of interest.

Copyright (C) 2017 Lebedeva, Westman, Borza, Beyer, Engedal, Aarsland, Selbaek and Haberg. This is an open-access article distributed under the terms of the Creative Commons Attribution License (CC BY). The use, distribution or reproduction in other forums is permitted, provided the original author(s) or licensor are credited and that the original publication in this journal is cited, in accordance with accepted academic practice. No use, distribution or reproduction is permitted which does not comply with these terms. 